\title{
On the solvability in the sense of sequences for some non-Fredholm operators
}

\author{
Vitali Vougalter and Vitaly Volpert
}

Communicated by Y. Charles Li, received April 7, 2014.

\begin{abstract}
We investigate solvability of certain linear nonhomogeneous elliptic equations and establish that under reasonable technical conditions the convergence in $L^{2}\left(\mathbb{R}^{d}\right)$ of their right sides yields the existence and the convergence in $H^{2}\left(\mathbb{R}^{d}\right)$ of the solutions. The problems involve the sums of second order differential operators without Fredholm property and we apply the methods of spectral and scattering theory for Schrödinger type operators analogously to our previous work [18].
\end{abstract}

\section{CONTENTS}

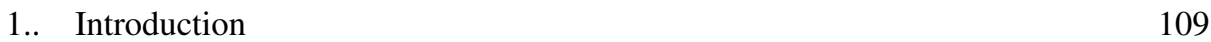

2.. Solvability in the sense of sequences in six dimensions $\quad 114$

3.. Solvability in the sense of sequences in $d+3$ dimensions 116

$\begin{array}{ll}\text { 4.. Auxiliary results } & 119\end{array}$

$\begin{array}{ll}\text { References } & 123\end{array}$

\section{1.. Introduction}

Let us consider the problem

$$
-\Delta u+V(x) u-a u=f,
$$

with $u \in E=H^{2}\left(\mathbb{R}^{d}\right)$ and $f \in F=L^{2}\left(\mathbb{R}^{d}\right), d \in \mathbb{N}, a$ is a constant and $V(x)$ is a real valued function decaying to 0 at infinity. If $a \geq 0$, then the origin belongs to the essential spectrum of the operator $A: E \rightarrow F$ corresponding to the left side of problem (1.1). Consequently, the operator fails to satisfy the Fredholm property. Its image is not closed, for $d>1$ the dimensions of its kernel and the codimension of its image are not finite. In the present article we will study certain properties of such operators. Let us note that elliptic equations containing operators without Fredholm property were studied extensively in recent years (see [17], [18], [19], [20], [21], [22], [23], [24], also [5]) along with their

Key words and phrases. Solvability conditions, non Fredholm operators, Sobolev spaces. 
potential applications to the theory of reaction-diffusion equations (see [7], [8]). Problems of that type arise also in the theory of embedded solitons of Nonlinear Schrödinger like equations (see e.g. [13], also [24]). In the particular case of $a=0$ the operator $A$ satisfies the Fredholm property in certain properly chosen weighted spaces (see [1], [2], [3], [4], [5]). However, the case of $a \neq 0$ is significantly different and the method developed in these works cannot be applied.

One of the crucial questions concerning equations involving operators without Fredholm property is their solvability. We will investigate the problem as follows. Suppose $f_{n}$ is a sequence of functions in the image of the operator $A$, such that $f_{n} \rightarrow f$ in $L^{2}\left(\mathbb{R}^{d}\right)$ as $n \rightarrow \infty$. Let $u_{n}$ be a sequence of functions from $H^{2}\left(\mathbb{R}^{d}\right)$ such that

$$
A u_{n}=f_{n}, n \in \mathbb{N} .
$$

Due to the fact that the operator $A$ is non Fredholm, the sequence $u_{n}$ can be bounded but not compact, it does contain a convergent subsequence. Let us call a sequence $u_{n}$ such that $A u_{n} \rightarrow f$ a solution in the sense of sequences of equation $A u=f$ (see [16]). If this sequence converges to a function $u_{0}$ in the norm of the space $E$, then $u_{0}$ is a solution of this equation. Solution in the sense of sequences is equivalent in this sense to the usual solution. However, in the case of operators without Fredholm property this convergence may not hold or it can occur in a certain weaker sense. In this case, a solution in the sense of sequences may not imply the existence of the usual solution. In the present article we determine sufficient conditions of equivalence of solutions in the sense of sequences to the usual solutions. In the other words, the conditions on sequences $f_{n}$ under which the corresponding sequences $u_{n}$ are strongly convergent.

In the first part of the work we consider the equation

$$
-\Delta_{x} u+V(x) u-\Delta_{y} u+U(y) u-a u=f(x, y), x, y \in \mathbb{R}^{3},
$$

where $a \geq 0$ is a constant and the right side is square integrable. Here $\Delta_{x}$ and $\Delta_{y}$ are the standard three dimensional Laplacians acting on $x$ and $y$ variables respectively. The potential functions $V(x)$ and $U(y)$ here are assumed to be shallow and short-range and precise assumptions on their behavior will be formulated below. The problem analogous to (1.2) involving a single non Fredholm Schrödinger operator in its left side was studied recently in the context of the solvability in the sense of sequences in [25]. Note that for each of the operators $-\Delta_{x}+V(x)$ and $-\Delta_{y}+U(y)$ on $L^{2}\left(\mathbb{R}^{3}\right)$ the essential spectrum fills the nonnegative semi-axis $[0, \infty$ ) (see e.g. [10]) such that the inverse of the whole operator in the left side of (1.2) from $L^{2}\left(\mathbb{R}^{6}\right)$ to $H^{2}\left(\mathbb{R}^{6}\right)$ is not bounded. We write down the corresponding sequence of iterated equations with $n \in \mathbb{N}$ and $a \geq 0$ as

$$
-\Delta_{x} u_{n}+V(x) u_{n}-\Delta_{y} u_{n}+U(y) u_{n}-a u_{n}=f_{n}(x, y), x, y \in \mathbb{R}^{3},
$$

with their right sides convergent to the right side of (1.2) in $L^{2}\left(\mathbb{R}^{6}\right)$ as $n \rightarrow \infty$. The inner product of two functions is denoted as

$$
(f(x), g(x))_{L^{2}\left(\mathbb{R}^{d}\right)}:=\int_{\mathbb{R}^{d}} f(x) \bar{g}(x) d x, d \in \mathbb{N}
$$

with a slight abuse of notations when these functions are not square integrable. Indeed, when $f(x) \in L^{1}\left(\mathbb{R}^{d}\right)$ and $g(x) \in L^{\infty}\left(\mathbb{R}^{d}\right)$, then evidently the integral above is well defined, like for example in the case of functions involved in the orthogonality relations of Theorems 2 and 3 below. In the space of $d$ dimensions for a certain

$$
A(x)=\left(A_{1}(x), \ldots, A_{d}(x)\right),
$$


the inner product $(f(x), A(x))_{L^{2}\left(\mathbb{R}^{d}\right)}$ is the vector with the coordinates

$$
\int_{\mathbb{R}^{d}} f(x) \bar{A}_{k}(x) d x, \quad 1 \leq k \leq d .
$$

Let us consider the standard space $H^{2}\left(\mathbb{R}^{d}\right)$ equipped with the norm

$$
\|u\|_{H^{2}\left(\mathbb{R}^{d}\right)}^{2}:=\|u\|_{L^{2}\left(\mathbb{R}^{d}\right)}^{2}+\|\Delta u\|_{L^{2}\left(\mathbb{R}^{d}\right)}^{2}, d \in \mathbb{N} .
$$

The sphere of radius $r>0$ in $\mathbb{R}^{d}$ centered at the origin will be designated as $S_{r}^{d}$.

Let us use the hat symbol to denote the standard Fourier transform

$$
\widehat{f}(p):=\frac{1}{(2 \pi)^{\frac{d}{2}}} \int_{\mathbb{R}^{d}} f(x) e^{-i p x} d x, p \in \mathbb{R}^{d}, d \in \mathbb{N} .
$$

In the second part of the article we will study the problem

$$
-\Delta_{x} u-\Delta_{y} u+U(y) u-a u=\phi(x, y), x \in \mathbb{R}^{d}, y \in \mathbb{R}^{3}, d \in \mathbb{N},
$$

with the right side square integrable and the constant $a \geq 0$. Here $\Delta_{x}$ and $\Delta_{y}$ are the standard Laplace operators acting on the variables $x$ and $y$ respectively. The corresponding sequence of iterated equations for $n \in \mathbb{N}$ will be given by

$$
-\Delta_{x} u_{n}-\Delta_{y} u_{n}+U(y) u_{n}-a u_{n}=\phi_{n}(x, y), x \in \mathbb{R}^{d}, y \in \mathbb{R}^{3}, a \geq 0,
$$

with their right sides converging to the right side of (1.6) in $L^{2}\left(\mathbb{R}^{d+3}\right)$ as $n \rightarrow \infty$. We formulate the technical conditions on the scalar potentials involved in the equations above. They will be analogous to those stated in Assumption 1.1 of [18] (see also [19], [20]).

Assumption 1. The potential functions $V(x), U(y): \mathbb{R}^{3} \rightarrow \mathbb{R}$ satisfy the bound

$$
|V(x)| \leq \frac{C}{1+|x|^{3.5+\delta}}, \quad|U(y)| \leq \frac{C}{1+|y|^{3.5+\delta}}
$$

with some $\delta>0$ and $x, y \in \mathbb{R}^{3}$ a.e. such that

$$
\begin{aligned}
& 4^{\frac{1}{9}} \frac{9}{8}(4 \pi)^{-\frac{2}{3}}\|V\|_{L^{\infty}\left(\mathbb{R}^{3}\right)}^{\frac{1}{9}}\|V\|_{L^{\frac{4}{3}\left(\mathbb{R}^{3}\right)}}^{\frac{8}{9}}<1, \\
& 4^{\frac{1}{9}} \frac{9}{8}(4 \pi)^{-\frac{2}{3}}\|U\|_{L^{\infty}\left(\mathbb{R}^{3}\right)}^{\frac{1}{9}}\|U\|_{L^{\frac{4}{3}\left(\mathbb{R}^{3}\right)}}^{\frac{8}{9}}<1
\end{aligned}
$$

and

$$
\sqrt{c_{H L S}}\|V\|_{L^{\frac{3}{2}}\left(\mathbb{R}^{3}\right)}<4 \pi, \quad \sqrt{c_{H L S}}\|U\|_{L^{\frac{3}{2}}\left(\mathbb{R}^{3}\right)}<4 \pi .
$$

Note that the rate of decay of the potential functions assumed above is sufficient for all their $L^{p}$ norms involved in the article to be finite. Here and below $C$ denotes a finite positive constant and $c_{H L S}$ given on p.98 of [12] is the constant in the Hardy-Littlewood-Sobolev inequality

$$
\left|\int_{\mathbb{R}^{3}} \int_{\mathbb{R}^{3}} \frac{f_{1}(x) f_{1}(y)}{|x-y|^{2}} d x d y\right| \leq c_{H L S}\left\|f_{1}\right\|_{L^{\frac{3}{2}}\left(\mathbb{R}^{3}\right)}^{2}, \quad f_{1} \in L^{\frac{3}{2}}\left(\mathbb{R}^{3}\right) .
$$

By virtue of Lemma 2.3 of [18], under Assumption 1 above on our potential functions, the operators $-\Delta_{x}+V(x)$ and $-\Delta_{y}+U(y)$ considered as acting in $L^{2}\left(\mathbb{R}^{3}\right)$ with domain $H^{2}\left(\mathbb{R}^{3}\right)$ are self-adjoint and unitarily equivalent to $-\Delta_{x}$ and $-\Delta_{y}$ respectively via the wave operators (see [11], [15])

$$
\Omega_{V}^{ \pm}:=s-\lim _{t \rightarrow \mp \infty} e^{i t\left(-\Delta_{x}+V(x)\right)} e^{i t \Delta_{x}}, \quad \Omega_{U}^{ \pm}:=s-\lim _{t \rightarrow \mp \infty} e^{i t\left(-\Delta_{y}+U(y)\right)} e^{i t \Delta_{y}},
$$


with the limits here understood in the strong $L^{2}$ sense (see e.g. [14] p.34, [6] p.90). Therefore the operators $-\Delta_{x}+V(x)$ and $-\Delta_{y}+U(y)$ possess only the essential spectra: $\sigma_{e s s}\left(-\Delta_{x}+V(x)\right)=[0, \infty)$ and $\sigma_{e s s}\left(-\Delta_{y}+U(y)\right)=[0, \infty)$. The functions of the continuous spectrum satisfy

$$
\begin{gathered}
{\left[-\Delta_{x}+V(x)\right] \varphi_{k}(x)=k^{2} \varphi_{k}(x), \quad k \in \mathbb{R}^{3},} \\
{\left[-\Delta_{y}+U(y)\right] \eta_{q}(y)=q^{2} \eta_{q}(y), \quad q \in \mathbb{R}^{3},}
\end{gathered}
$$

in the integral formulation the Lippmann-Schwinger equations for the perturbed plane waves (see e.g. [14] p.98)

$$
\begin{aligned}
& \varphi_{k}(x)=\frac{e^{i k x}}{(2 \pi)^{\frac{3}{2}}}-\frac{1}{4 \pi} \int_{\mathbb{R}^{3}} \frac{e^{i|k||x-y|}}{|x-y|}\left(V \varphi_{k}\right)(y) d y . \\
& \eta_{q}(y)=\frac{e^{i q y}}{(2 \pi)^{\frac{3}{2}}}-\frac{1}{4 \pi} \int_{\mathbb{R}^{3}} \frac{e^{i|q||y-z|}}{|y-z|}\left(U \eta_{q}\right)(z) d z,
\end{aligned}
$$

and the orthogonality conditions

$$
\begin{gathered}
\left(\varphi_{k}(x), \varphi_{l}(x)\right)_{L^{2}\left(\mathbb{R}^{3}\right)}=\delta(k-l), \quad k, l \in \mathbb{R}^{3}, \\
\left(\eta_{q}(y), \eta_{m}(y)\right)_{L^{2}\left(\mathbb{R}^{3}\right)}=\delta(q-m), \quad q, m \in \mathbb{R}^{3} .
\end{gathered}
$$

The $\delta$ symbol in the formulas above denotes the standard Dirac delta measure. By virtue of the Spectral theorem, the products of $\varphi_{k}(x)$ and $\eta_{q}(y)$ form a complete system in $L^{2}\left(\mathbb{R}^{6}\right)$. We denote the generalized Fourier transform with respect to these products using the double tilde symbol as

$$
\tilde{\tilde{f}}(k, q):=\left(f(x, y), \varphi_{k}(x) \eta_{q}(y)\right)_{L^{2}\left(\mathbb{R}^{6}\right)}, k, q \in \mathbb{R}^{3} .
$$

Clearly, (1.16) is a unitary transform on $L^{2}\left(\mathbb{R}^{6}\right)$, which can be easily seen via orthogonality relations (1.14) and (1.15). We designate the integral operators involved in (1.12) and (1.13) as

$$
\begin{array}{ll}
(Q \varphi)(x):=-\frac{1}{4 \pi} \int_{\mathbb{R}^{3}} \frac{e^{i|k||x-y|}}{|x-y|}(V \varphi)(y) d y, \quad \varphi \in L^{\infty}\left(\mathbb{R}^{3}\right), \\
(P \eta)(y):=-\frac{1}{4 \pi} \int_{\mathbb{R}^{3}} \frac{e^{i|q||y-z|}}{|y-z|}(U \eta)(z) d z, \quad \eta \in L^{\infty}\left(\mathbb{R}^{3}\right),
\end{array}
$$

and consider $Q, P: L^{\infty}\left(\mathbb{R}^{3}\right) \rightarrow L^{\infty}\left(\mathbb{R}^{3}\right)$. Under Assumption 1, by virtue of Lemma 2.1 of [18] the operator norms $\|Q\|_{\infty}<1$ and $\|P\|_{\infty}<1$, in fact they are bounded above by the left sides of inequalities (1.8) and (1.9) respectively, which are quantities independent of $k$ and $q$, expressed in terms of the corresponding $L^{p}\left(\mathbb{R}^{3}\right)$ norms of the scalar potential functions $V(x)$ and $U(y)$. In the context of the studies of equations (1.6) and (1.7), we will be using products of Fourier harmonics $\frac{e^{i k x}}{(2 \pi)^{\frac{d}{2}}}, k \in \mathbb{R}^{d}$ and perturbed plane waves $\eta_{q}(y)$ forming a complete system in $L^{2}\left(\mathbb{R}^{d+3}\right)$, such that the generalized Fourier transform with respect to these products is given by

$$
\tilde{\hat{f}}(k, q):=\left(f(x, y), \frac{e^{i k x}}{(2 \pi)^{\frac{d}{2}}} \eta_{q}(y)\right)_{L^{2}\left(\mathbb{R}^{d+3}\right)}, k \in \mathbb{R}^{d}, q \in \mathbb{R}^{3} .
$$

Obviously, (1.17) is a unitary transform on $L^{2}\left(\mathbb{R}^{d+3}\right)$, which can be trivially obtained via orthogonality relation (1.15). We formulate our main statements. 
Theorem 2. Let Assumption 1 hold, $x, y \in \mathbb{R}^{3}, n \in \mathbb{N}$ and $f_{n}(x, y) \in L^{2}\left(\mathbb{R}^{6}\right)$, such that $f_{n}(x, y) \rightarrow f(x, y)$ in $L^{2}\left(\mathbb{R}^{6}\right)$ as $n \rightarrow \infty$. Assume also that $|x| f_{n}(x, y) \in L^{1}\left(\mathbb{R}^{6}\right)$ and $|y| f_{n}(x, y) \in L^{1}\left(\mathbb{R}^{6}\right)$, such that

$$
|x| f_{n}(x, y) \rightarrow|x| f(x, y), \quad|y| f_{n}(x, y) \rightarrow|y| f(x, y)
$$

in $L^{1}\left(\mathbb{R}^{6}\right)$ as $n \rightarrow \infty$.

a) When $a>0$ let the orthogonality relations

$$
\left(f_{n}(x, y), \varphi_{k}(x) \eta_{q}(y)\right)_{L^{2}\left(\mathbb{R}^{6}\right)}=0,(k, q) \in S_{\sqrt{a}}^{6} \text { a.e. }
$$

hold for all $n \in \mathbb{N}$. Then equations (1.2) and (1.3) have unique solutions $u(x, y) \in$ $H^{2}\left(\mathbb{R}^{6}\right)$ and $u_{n}(x, y) \in H^{2}\left(\mathbb{R}^{6}\right)$ respectively, such that $u_{n}(x, y) \rightarrow u(x, y)$ in $H^{2}\left(\mathbb{R}^{6}\right)$ as $n \rightarrow \infty$.

b) When $a=0$ let $u_{n}(x, y) \in H^{2}\left(\mathbb{R}^{6}\right)$ be the unique solution of problem (1.3), $n \in \mathbb{N}$ and equation (1.2) possesses a unique solution $u(x, y) \in H^{2}\left(\mathbb{R}^{6}\right)$. Then $u_{n}(x, y) \rightarrow$ $u(x, y)$ in $H^{2}\left(\mathbb{R}^{6}\right)$ as $n \rightarrow \infty$.

Note that according to the part b) of Theorem 3 of [19] (see also [21]) for our case b) when $a=0$ the orthogonality conditions are not needed as distinct from case a). Similarly in the case b) of the theorem below we do not require any orthogonality relations when the $x$ dimension of the problem is at least two (see Theorem 6 of [19], also [21]).

Theorem 3. Let Assumption 1 hold for the potential function $U(y), y \in \mathbb{R}^{3}, x \in$ $\mathbb{R}^{d}, d \geq 1, n \in \mathbb{N}$ and $\phi_{n}(x, y) \in L^{2}\left(\mathbb{R}^{d+3}\right)$, such that $\phi_{n}(x, y) \rightarrow \phi(x, y)$ in $L^{2}\left(\mathbb{R}^{d+3}\right)$ as $n \rightarrow \infty$. Suppose as well that

$$
|x| \phi_{n}(x, y) \in L^{1}\left(\mathbb{R}^{d+3}\right), \quad|y| \phi_{n}(x, y) \in L^{1}\left(\mathbb{R}^{d+3}\right),
$$

such that

$$
|x| \phi_{n}(x, y) \rightarrow|x| \phi(x, y), \quad|y| \phi_{n}(x, y) \rightarrow|y| \phi(x, y)
$$

in $L^{1}\left(\mathbb{R}^{d+3}\right)$ as $n \rightarrow \infty$.

a) When $a>0$ let the orthogonality conditions

$$
\left(\phi_{n}(x, y), \frac{e^{i k x}}{(2 \pi)^{\frac{d}{2}}} \eta_{q}(y)\right)_{L^{2}\left(\mathbb{R}^{d+3}\right)}=0,(k, q) \in S_{\sqrt{a}}^{d+3} \text { a.e. }
$$

be valid for all $n \in \mathbb{N}$. Then equations (1.6) and (1.7) admit unique solutions $u(x, y) \in$ $H^{2}\left(\mathbb{R}^{d+3}\right)$ and $u_{n}(x, y) \in H^{2}\left(\mathbb{R}^{d+3}\right)$ respectively, such that $u_{n}(x, y) \rightarrow u(x, y)$ in $H^{2}\left(\mathbb{R}^{d+3}\right)$ as $n \rightarrow \infty$.

b) When $a=0$ and the dimension $d=1$ let the orthogonality relations

$$
\left(\phi_{n}(x, y), \eta_{0}(y)\right)_{L^{2}\left(\mathbb{R}^{4}\right)}=0
$$

hold for all $n \in \mathbb{N}$ and no orthogonality conditions are assumed in dimensions $d \geq 2$. Then problems (1.6) and (1.7) possess unique solutions $u(x, y) \in H^{2}\left(\mathbb{R}^{d+3}\right)$ and $u_{n}(x, y) \in$ $H^{2}\left(\mathbb{R}^{d+3}\right)$ respectively, such that $u_{n}(x, y) \rightarrow u(x, y)$ in $H^{2}\left(\mathbb{R}^{d+3}\right)$ as $n \rightarrow \infty$.

Note that (1.18), (1.19) and (1.20) are the orthogonality conditions involving the functions of the continuous spectrum of our Schrödinger operators, as distinct from the Limiting Absorption Principle in which one needs to orthogonalize to the standard Fourier harmonics (see e.g. Lemma 2.3 and Proposition 2.4 of [9]). We proceed with proving our first main result. 


\section{2.. Solvability in the sense of sequences in six dimensions}

Proof of Theorem 2. Four our equations (1.2) and (1.3) the solvability in $L^{2}\left(\mathbb{R}^{6}\right)$ follows from Theorem 3 of [19]. Since their right sides are square integrable and the scalar potentials $V(x)$ and $U(y)$ involved there are bounded as assumed, we will have the existence of their unique solutions in $H^{2}\left(\mathbb{R}^{6}\right)$ as well.

Let us first start with the case b) of the theorem when the constant $a=0$, such that by means of the part b) of Theorem 3 of [19] the orthogonality conditions are not needed here. Then let $u_{n}(x, y) \in H^{2}\left(\mathbb{R}^{6}\right), n \in \mathbb{N}$ and $u(x, y) \in H^{2}\left(\mathbb{R}^{6}\right)$ be the unique solutions of problems (1.3) and (1.2) respectively.

By applying the generalized Fourier transform (1.16) to both sides of problems (1.2) and (1.3) with $k, q \in \mathbb{R}^{3}$ we arrive at

$$
\tilde{\tilde{u}}(k, q)=\frac{\tilde{\tilde{f}}(k, q)}{k^{2}+q^{2}}, \quad \tilde{\tilde{u}}_{n}(k, q)=\frac{\tilde{\tilde{f}}_{n}(k, q)}{k^{2}+q^{2}}, \quad n \in \mathbb{N} .
$$

Let us write their difference as

$$
\begin{gathered}
\tilde{\tilde{u}}_{n}(k, q)-\tilde{\tilde{u}}(k, q)=\frac{\tilde{\tilde{f}}_{n}(k, q)-\tilde{\tilde{f}}(k, q)}{k^{2}+q^{2}} \chi_{\left\{(k, q) \in \mathbb{R}^{6}: k^{2}+q^{2} \leq 1\right\}}+ \\
+\frac{\tilde{\tilde{f}}(k, q)-\tilde{\tilde{f}}(k, q)}{k^{2}+q^{2}} \chi_{\left\{(k, q) \in \mathbb{R}^{6}: k^{2}+q^{2}>1\right\}} .
\end{gathered}
$$

Here and below $\chi_{A}$ will denote the characteristic function of a set $A \subseteq \mathbb{R}^{d}$ and $A^{c}$ will stand for the complement of this set. Evidently the second term in the right side of (2.1) can be estimated from above in the absolute value by $\left|\tilde{\tilde{f}}_{n}(k, q)-\tilde{\tilde{f}}(k, q)\right|$ and therefore, in the $L^{2}\left(\mathbb{R}^{6}\right)$ norm by $\left\|f_{n}-f\right\|_{L^{2}\left(\mathbb{R}^{6}\right)} \rightarrow 0, n \rightarrow \infty$ as assumed. By means of our assumptions and via the Schwarz inequality we have $f_{n}(x, y) \in L^{1}\left(\mathbb{R}^{6}\right), n \in \mathbb{N}$. We estimate the norm from above using again the Schwarz inequality as

$$
\begin{aligned}
& \left\|f_{n}-f\right\|_{L^{1}\left(\mathbb{R}^{6}\right)} \leq \sqrt{\int_{x^{2}+y^{2} \leq 1}\left|f_{n}(x, y)-f(x, y)\right|^{2} d x d y} \sqrt{\int_{x^{2}+y^{2} \leq 1} d x d y+} \\
& +\int_{x^{2}+y^{2}>1}\left|f_{n}(x, y)-f(x, y)\right| \sqrt{x^{2}+y^{2}} d x d y \leq C\left\|f_{n}-f\right\|_{L^{2}\left(\mathbb{R}^{6}\right)}+ \\
& \quad+\left\||x| f_{n}-|x| f\right\|_{L^{1}\left(\mathbb{R}^{6}\right)}+\left\||y| f_{n}-|y| f\right\|_{L^{1}\left(\mathbb{R}^{6}\right)} \rightarrow 0, \quad n \rightarrow \infty
\end{aligned}
$$

due to the assumptions of the theorem. Hence

$$
f_{n}(x, y) \rightarrow f(x, y) \quad \text { in } \quad L^{1}\left(\mathbb{R}^{6}\right), \quad n \rightarrow \infty .
$$

Let us express

$$
\begin{gathered}
\tilde{\tilde{f}}_{n}(k, q)=\tilde{\tilde{f}}_{n}(0)+\int_{0}^{\sqrt{k^{2}+q^{2}}} \frac{\partial \tilde{\tilde{f}}_{n}}{\partial s}(s, \omega) d s, \\
\tilde{\tilde{f}}(k, q)=\tilde{\tilde{f}}(0)+\int_{0}^{\sqrt{k^{2}+q^{2}}} \frac{\partial \tilde{\tilde{f}}}{\partial s}(s, \omega) d s .
\end{gathered}
$$

Here and below $\omega$ denotes the angle variables on the sphere. This enables us to write the first term in the right side of (2.1) as

$$
\frac{\tilde{\tilde{f}}_{n}(0)-\tilde{\tilde{f}}(0)}{k^{2}+q^{2}} \chi_{\left\{(k, q) \in \mathbb{R}^{6}: k^{2}+q^{2} \leq 1\right\}}+
$$




$$
+\frac{\int_{0}^{\sqrt{k^{2}+q^{2}}} \frac{\partial}{\partial s}\left[\tilde{\tilde{f}}_{n}(s, \omega)-\tilde{\tilde{f}}(s, \omega)\right] d s}{k^{2}+q^{2}} \chi_{\left\{(k, q) \in \mathbb{R}^{6}: k^{2}+q^{2} \leq 1\right\}} .
$$

By means of Corollary 2.2 of [18] (see also [19]) under our assumptions for $k, q \in \mathbb{R}^{3}$ we have $\varphi_{k}(x), \eta_{q}(y) \in L^{\infty}\left(\mathbb{R}^{3}\right)$ due to the inequalities

$$
\left\|\varphi_{k}(x)\right\|_{L^{\infty}\left(\mathbb{R}^{3}\right)} \leq \frac{1}{1-\|Q\|_{\infty}} \frac{1}{(2 \pi)^{\frac{3}{2}}}, \quad\left\|\eta_{q}(y)\right\|_{L^{\infty}\left(\mathbb{R}^{3}\right)} \leq \frac{1}{1-\|P\|_{\infty}} \frac{1}{(2 \pi)^{\frac{3}{2}}} .
$$

This enables us to estimate from above in the absolute value the first term in (2.3) by

$$
\frac{1}{(2 \pi)^{3}} \frac{1}{1-\|Q\|_{\infty}} \frac{1}{1-\|P\|_{\infty}}\left\|f_{n}-f\right\|_{L^{1}\left(\mathbb{R}^{6}\right)} \frac{\chi_{\left\{(k, q) \in \mathbb{R}^{6}: k^{2}+q^{2} \leq 1\right\}}}{k^{2}+q^{2}},
$$

which clearly yields the upper bound for it in the $L^{2}\left(\mathbb{R}^{6}\right)$ norm by

$$
C \frac{1}{1-\|Q\|_{\infty}} \frac{1}{1-\|P\|_{\infty}}\left\|f_{n}-f\right\|_{L^{1}\left(\mathbb{R}^{6}\right)} \rightarrow 0, \quad n \rightarrow \infty
$$

by virtue of (2.2) under our assumptions. Then we estimate from above in the absolute value the second term in (2.3) as

$$
\left\|\left(\nabla_{k}+\nabla_{q}\right)\left[\tilde{\tilde{f}}_{n}(k, q)-\tilde{\tilde{f}}(k, q)\right]\right\|_{L^{\infty}\left(\mathbb{R}^{6}\right)} \frac{\chi_{\left\{(k, q) \in \mathbb{R}^{6}: k^{2}+q^{2} \leq 1\right\}}}{\sqrt{k^{2}+q^{2}}},
$$

which gives us the upper bound for it in the $L^{2}\left(\mathbb{R}^{6}\right)$ norm as

$$
C\left\|\left(\nabla_{k}+\nabla_{q}\right)\left[\tilde{\tilde{f}}_{n}(k, q)-\tilde{\tilde{f}}(k, q)\right]\right\|_{L^{\infty}\left(\mathbb{R}^{6}\right)} \rightarrow 0, \quad n \rightarrow \infty
$$

due to the part a) of Lemma 5 below. Here and further down notations $\nabla_{k}$ and $\nabla_{q}$ stand for the gradients taken with respect to $k$ and $q$ variables respectively. Hence

$$
\left\|u_{n}-u\right\|_{L^{2}\left(\mathbb{R}^{6}\right)}=\left\|\tilde{\tilde{u}}_{n}(k, q)-\tilde{\tilde{u}}(k, q)\right\|_{L^{2}\left(\mathbb{R}^{6}\right)} \rightarrow 0, \quad n \rightarrow \infty .
$$

By means of the part a) of Lemma 4 below, $u_{n}(x, y) \rightarrow u(x, y)$ in $H^{2}\left(\mathbb{R}^{6}\right)$ as $n \rightarrow \infty$, which completes the proof of part b) of the theorem.

Then we turn our attention to establishing the results of the part a) of the theorem when $a>0$, such that orthogonality conditions (1.18) hold. By means of part a) of Theorem 3 of [19], equation (1.3) admits a unique solution $u_{n}(x, y) \in H^{2}\left(\mathbb{R}^{6}\right), n \in \mathbb{N}$. For $(k, q) \in S_{\sqrt{a}}^{6}$ a.e., using (2.4) we easily estimate

$$
\left|\left(f(x, y), \varphi_{k}(x) \eta_{q}(y)\right)_{L^{2}\left(\mathbb{R}^{6}\right)}\right|=\left|\left(f(x, y)-f_{n}(x, y), \varphi_{k}(x) \eta_{q}(y)\right)_{L^{2}\left(\mathbb{R}^{6}\right)}\right|
$$

from above by

$$
\frac{1}{(2 \pi)^{3}} \frac{1}{1-\|Q\|_{\infty}} \frac{1}{1-\|P\|_{\infty}}\left\|f_{n}-f\right\|_{L^{1}\left(\mathbb{R}^{6}\right)} \rightarrow 0, \quad n \rightarrow \infty
$$

by means of (2.2). Hence in the limit the orthogonality relation

$$
\left(f(x, y), \varphi_{k}(x) \eta_{q}(y)\right)_{L^{2}\left(\mathbb{R}^{6}\right)}=0, \quad(k, q) \in S_{\sqrt{a}}^{6} \quad \text { a.e. }
$$

is valid. By virtue of the part a) of Theorem 3 of [19], the limiting problem (1.2) has a unique solution $u(x, y) \in H^{2}\left(\mathbb{R}^{6}\right)$ when $a>0$. Let us apply the generalized Fourier transform (1.16) to both sides of equations (1.2) and (1.3). We easily arrive at

$$
\tilde{\tilde{u}}(k, q)=\frac{\tilde{\tilde{f}}(k, q)}{k^{2}+q^{2}-a}, \quad \tilde{\tilde{u}}_{n}(k, q)=\frac{\tilde{\tilde{f}}_{n}(k, q)}{k^{2}+q^{2}-a}, \quad n \in \mathbb{N} .
$$

For technical purposes, let us introduce a layer in the space of six dimensions as

$$
A_{\sigma}:=\left\{(k, q) \in \mathbb{R}^{6} \mid \sqrt{a}-\sigma \leq \sqrt{k^{2}+q^{2}} \leq \sqrt{a}+\sigma\right\}, \quad 0<\sigma<\sqrt{a}
$$


and express the difference $\tilde{\tilde{u}}(k, q)-\tilde{\tilde{u}}_{n}(k, q)$ as

$$
\frac{\tilde{\tilde{f}}(k, q)-\tilde{\tilde{f}}_{n}(k, q)}{k^{2}+q^{2}-a} \chi_{A_{\sigma}}+\frac{\tilde{\tilde{f}}(k, q)-\tilde{\tilde{f}}_{n}(k, q)}{k^{2}+q^{2}-a} \chi_{A_{\sigma}^{c}} .
$$

The second term in (2.6) can be trivially estimated from above in the absolute value by

$$
\frac{\left|\tilde{\tilde{f}}(k, q)-\tilde{\tilde{f}}_{n}(k, q)\right|}{\sqrt{a} \sigma} \chi_{A_{\sigma}^{c}},
$$

which yields the following upper bound on it in the $L^{2}\left(\mathbb{R}^{6}\right)$ norm

$$
\frac{\left\|\tilde{\tilde{f}}(k, q)-\tilde{\tilde{f}}_{n}(k, q)\right\|_{L^{2}\left(\mathbb{R}^{6}\right)}}{\sqrt{a} \sigma}=\frac{\left\|f-f_{n}\right\|_{L^{2}\left(\mathbb{R}^{6}\right)}}{\sqrt{a} \sigma} \rightarrow 0, \quad n \rightarrow \infty
$$

according to one of the assumptions of the theorem. We will use the identities

$$
\begin{gathered}
\tilde{\tilde{f}}_{n}(k, q)=\tilde{\tilde{f}}_{n}(\sqrt{a}, \omega)+\int_{\sqrt{a}}^{\sqrt{k^{2}+q^{2}}} \frac{\partial \tilde{\tilde{f}}_{n}}{\partial s}(s, \omega) d s, \\
\tilde{\tilde{f}}(k, q)=\tilde{\tilde{f}}(\sqrt{a}, \omega)+\int_{\sqrt{a}}^{\sqrt{k^{2}+q^{2}}} \frac{\partial \tilde{\tilde{f}}}{\partial s}(s, \omega) d s .
\end{gathered}
$$

Note that by virtue of orthogonality conditions (1.18) and (2.5), the first terms in the right sides of the formulas above $\tilde{\tilde{f}}_{n}(\sqrt{a}, \omega)$ and $\tilde{\tilde{f}}(\sqrt{a}, \omega)$ vanish for $(k, q) \in S_{\sqrt{a}}^{6}$ a.e. This enables us to estimate from above in the absolute value the first term in (2.6) by

$$
\frac{\left\|\left(\nabla_{k}+\nabla_{q}\right)\left[\tilde{\tilde{f}}(k, q)-\tilde{\tilde{f}}_{n}(k, q)\right]\right\|_{L^{\infty}\left(\mathbb{R}^{6}\right)}}{\sqrt{a}} \chi_{A_{\sigma}},
$$

which clearly implies the upper bound on it in the $L^{2}\left(\mathbb{R}^{6}\right)$ norm given by

$$
C\left\|\left(\nabla_{k}+\nabla_{q}\right)\left[\tilde{\tilde{f}}(k, q)-\tilde{\tilde{f}}_{n}(k, q)\right]\right\|_{L^{\infty}\left(\mathbb{R}^{6}\right)} \rightarrow 0, \quad n \rightarrow \infty
$$

according to the part a) of Lemma 5 below. Thus, we arrive at

$$
\left\|u-u_{n}\right\|_{L^{2}\left(\mathbb{R}^{6}\right)}=\left\|\tilde{\tilde{u}}(k, q)-\tilde{\tilde{u}}_{n}(k, q)\right\|_{L^{2}\left(\mathbb{R}^{6}\right)} \rightarrow 0, \quad n \rightarrow \infty,
$$

such that $u_{n}(x, y) \rightarrow u(x, y)$ in $L^{2}\left(\mathbb{R}^{6}\right)$ as $n \rightarrow \infty$. By virtue of the part a) of Lemma 4 below, we obtain $u_{n}(x, y) \rightarrow u(x, y)$ in $H^{2}\left(\mathbb{R}^{6}\right)$ as $n \rightarrow \infty$, which completes the proof of the part a) of the theorem.

\section{Solvability in the sense of sequences in $\mathrm{d}+3$ dimensions}

Proof of Theorem 3. For our problems (1.6) and (1.7) the solvability in $L^{2}\left(\mathbb{R}^{d+3}\right)$ stems from Theorem 6 of [19]. Due to the fact that the right sides of these equations belong to $L^{2}\left(\mathbb{R}^{d+3}\right)$ and the potential function $U(y)$ is bounded as assumed, we will have then the existence of their unique solutions in $H^{2}\left(\mathbb{R}^{d+3}\right)$.

The conditions of our theorem along with the Schwarz inequality imply that $\phi_{n}(x, y) \in$ $L^{1}\left(\mathbb{R}^{d+3}\right)$. We use the Schwarz inequality again to obtain the upper bound for the norm $\left\|\phi_{n}-\phi\right\|_{L^{1}\left(\mathbb{R}^{d+3}\right)}$ as

$$
\begin{gathered}
\sqrt{\int_{x^{2}+y^{2} \leq 1}\left|\phi_{n}(x, y)-\phi(x, y)\right|^{2} d x d y} \sqrt{\int_{x^{2}+y^{2} \leq 1} d x d y+} \\
+\int_{x^{2}+y^{2}>1} \sqrt{x^{2}+y^{2}}\left|\phi_{n}(x, y)-\phi(x, y)\right| d x d y \leq C\left\|\phi_{n}-\phi\right\|_{L^{2}\left(\mathbb{R}^{d+3}\right)}+
\end{gathered}
$$




$$
+|||x| \phi_{n}-|x| \phi\left\|_{L^{1}\left(\mathbb{R}^{d+3}\right)}+\right\||y| \phi_{n}-|y| \phi \|_{L^{1}\left(\mathbb{R}^{d+3}\right)} \rightarrow 0, \quad n \rightarrow \infty
$$

by virtue of the assumptions of our theorem. Thus for $d \geq 1$

$$
\phi_{n}(x, y) \rightarrow \phi(x, y) \quad \text { in } \quad L^{1}\left(\mathbb{R}^{d+3}\right), \quad n \rightarrow \infty .
$$

First we start with the case b) of the theorem, such that the constant $a=0$. When the dimension $d=1$, we have the orthogonality condition (1.20) which according to the part $\mathrm{b}$ ) of Theorem 6 of [19] guarantees us the existence of a unique solution $u_{n}(x, y) \in H^{2}\left(\mathbb{R}^{4}\right)$ of problem (1.7) with $n \in \mathbb{N}$. Using (2.4), we estimate from above

$$
\left|\left(\phi(x, y), \eta_{0}(y)\right)_{L^{2}\left(\mathbb{R}^{4}\right)}\right|=\left|\left(\phi(x, y)-\phi_{n}(x, y), \eta_{0}(y)\right)_{L^{2}\left(\mathbb{R}^{4}\right)}\right|
$$

as

$$
\frac{1}{(2 \pi)^{\frac{3}{2}}} \frac{1}{1-\|P\|_{\infty}}\left\|\phi_{n}-\phi\right\|_{L^{1}\left(\mathbb{R}^{4}\right)} \rightarrow 0, \quad n \rightarrow \infty
$$

via (3.1), such that in the limit we have the orthogonality relation

$$
\left(\phi(x, y), \eta_{0}(y)\right)_{L^{2}\left(\mathbb{R}^{4}\right)}=0 .
$$

Then by virtue of the part b) of Theorem 6 of [19] under our assumptions, the limiting equation (1.6) admits a unique solution $u(x, y) \in H^{2}\left(\mathbb{R}^{4}\right)$ as well.

In higher dimensions $d \geq 2$ according to the part c) of Theorem 6 of [19], no orthogonality conditions are needed, such that under our conditions problems (1.6) and (1.7) possess unique solutions $u(x, y) \in H^{2}\left(\mathbb{R}^{d+3}\right)$ and $u_{n}(x, y) \in H^{2}\left(\mathbb{R}^{d+3}\right)$ respectively. Let us apply the generalized Fourier transform (1.17) to both sides of (1.6) and (1.7) to obtain

$$
\tilde{\hat{u}}(k, q)=\frac{\tilde{\hat{\phi}}(k, q)}{k^{2}+q^{2}}, \quad \tilde{\hat{u}}_{n}(k, q)=\frac{\tilde{\hat{\phi}}_{n}(k, q)}{k^{2}+q^{2}}, \quad n \in \mathbb{N} .
$$

We write the difference $\tilde{\hat{u}}_{n}(k, q)-\tilde{\hat{u}}(k, q)$ as

$$
\frac{\tilde{\hat{\phi}}_{n}(k, q)-\tilde{\hat{\phi}}(k, q)}{k^{2}+q^{2}} \chi_{\left\{(k, q) \in \mathbb{R}^{d+3}: k^{2}+q^{2} \leq 1\right\}}+\frac{\tilde{\hat{\phi}}_{n}(k, q)-\tilde{\hat{\phi}}(k, q)}{k^{2}+q^{2}} \chi_{\left\{(k, q) \in \mathbb{R}^{d+3}: k^{2}+q^{2}>1\right\}} .
$$

Clearly, the second term in (3.3) can be easily estimated from above in the absolute value by $\left|\tilde{\hat{\phi}}_{n}(k, q)-\tilde{\hat{\phi}}(k, q)\right|$ and therefore in the $L^{2}\left(\mathbb{R}^{d+3}\right)$ norm by

$$
\left\|\tilde{\hat{\phi}}_{n}(k, q)-\tilde{\hat{\phi}}(k, q)\right\|_{L^{2}\left(\mathbb{R}^{d+3}\right)}=\left\|\phi_{n}-\phi\right\|_{L^{2}\left(\mathbb{R}^{d+3}\right)} \rightarrow 0, \quad n \rightarrow \infty
$$

due to one of the assumptions of our theorem. Let us express

$$
\begin{gathered}
\tilde{\hat{\phi}}(k, q)=\tilde{\hat{\phi}}(0)+\int_{0}^{\sqrt{k^{2}+q^{2}}} \frac{\partial \hat{\hat{\phi}}}{\partial s}(s, \omega) d s, \\
\tilde{\hat{\phi}}_{n}(k, q)=\tilde{\hat{\phi}}_{n}(0)+\int_{0}^{\sqrt{k^{2}+q^{2}}} \frac{\partial \tilde{\hat{\phi}}_{n}}{\partial s}(s, \omega) d s,
\end{gathered}
$$

which enables us to write the first term in (3.3) as

$$
\begin{gathered}
\frac{\tilde{\hat{\phi}}_{n}(0)-\tilde{\hat{\phi}}(0)}{k^{2}+q^{2}} \chi_{\left\{(k, q) \in \mathbb{R}^{d+3}: k^{2}+q^{2} \leq 1\right\}}+ \\
+\frac{\int_{0}^{\sqrt{k^{2}+q^{2}}} \frac{\partial}{\partial s}\left[\tilde{\hat{\phi}}_{n}(s, \omega)-\tilde{\hat{\phi}}(s, \omega)\right] d s}{k^{2}+q^{2}} \chi_{\left\{(k, q) \in \mathbb{R}^{d+3}: k^{2}+q^{2} \leq 1\right\}} .
\end{gathered}
$$


We easily estimate the second term in (3.4) from above in the absolute value by

$$
\left\|\left(\nabla_{k}+\nabla_{q}\right)\left[\tilde{\hat{\phi}}_{n}(k, q)-\tilde{\hat{\phi}}(k, q)\right]\right\|_{L^{\infty}\left(\mathbb{R}^{d+3}\right)} \frac{\chi_{\left\{(k, q) \in \mathbb{R}^{d+3}: k^{2}+q^{2} \leq 1\right\}}}{\sqrt{k^{2}+q^{2}}},
$$

which trivially implies the upper bound for it in the $L^{2}\left(\mathbb{R}^{d+3}\right)$ norm as

$$
C\left\|\left(\nabla_{k}+\nabla_{q}\right)\left[\tilde{\hat{\phi}}_{n}(k, q)-\tilde{\hat{\phi}}(k, q)\right]\right\|_{L^{\infty}\left(\mathbb{R}^{d+3}\right)} \rightarrow 0, \quad n \rightarrow \infty
$$

by virtue of the part b) of Lemma 5 below. To investigate the first term in (3.4), we recall that for $n \in \mathbb{N}$

$$
\tilde{\hat{\phi}}_{n}(0)=\frac{1}{(2 \pi)^{\frac{d}{2}}}\left(\phi_{n}(x, y), \eta_{0}(y)\right)_{L^{2}\left(\mathbb{R}^{d+3}\right)}, \quad \tilde{\hat{\phi}}(0)=\frac{1}{(2 \pi)^{\frac{d}{2}}}\left(\phi(x, y), \eta_{0}(y)\right)_{L^{2}\left(\mathbb{R}^{d+3}\right)} .
$$

Hence when the dimension $d=1$, we have $\tilde{\hat{\phi}}_{n}(0)=0, n \in \mathbb{N}$ and $\tilde{\hat{\phi}}(0)=0$ by virtue of orthogonality relations (1.20) and (3.2) respectively, such that the first term in (3.4) then vanishes. For higher dimensions $d \geq 2$, using (3.5) and (2.4), we estimate the first term in (3.4) from above in the absolute value by

$$
\frac{1}{(2 \pi)^{\frac{d+3}{2}}} \frac{1}{1-\|P\|_{\infty}}\left\|\phi_{n}-\phi\right\|_{L^{1}\left(\mathbb{R}^{d+3}\right)} \frac{\chi_{\left\{(k, q) \in \mathbb{R}^{d+3}: k^{2}+q^{2} \leq 1\right\}}}{k^{2}+q^{2}},
$$

which gives us the upper bound for it in the $L^{2}\left(\mathbb{R}^{d+3}\right)$ norm as

$$
C \frac{1}{1-\|P\|_{\infty}}\left\|\phi_{n}-\phi\right\|_{L^{1}\left(\mathbb{R}^{d+3}\right)} \rightarrow 0, \quad n \rightarrow \infty
$$

due to (3.1). Therefore, when $a=0$, we have

$$
\left\|u_{n}(x, y)-u(x, y)\right\|_{L^{2}\left(\mathbb{R}^{d+3}\right)}=\left\|\tilde{\hat{u}}_{n}(k, q)-\tilde{\hat{u}}(k, q)\right\|_{L^{2}\left(\mathbb{R}^{d+3}\right)} \rightarrow 0, \quad n \rightarrow \infty,
$$

By means of the part b) of Lemma 4 below $u_{n}(x, y) \rightarrow u(x, y)$ in $H^{2}\left(\mathbb{R}^{d+3}\right)$ as $n \rightarrow \infty$, which completes the proof of the part b) of the theorem.

We conclude the argument with establishing the results of the part a), when the constant $a>0$. Orthogonality conditions (1.19) via the part a) of Theorem 6 of [19] imply that problem (1.7) admits a unique solution $u_{n}(x, y) \in H^{2}\left(\mathbb{R}^{d+3}\right), n \in \mathbb{N}$. For $(k, q) \in S_{\sqrt{a}}^{d+3}$ a.e., using (2.4), we estimate the expression

$$
\left|\left(\phi(x, y), \frac{e^{i k x}}{(2 \pi)^{\frac{d}{2}}} \eta_{q}(y)\right)_{L^{2}\left(\mathbb{R}^{d+3}\right)}\right|=\left|\left(\phi(x, y)-\phi_{n}(x, y), \frac{e^{i k x}}{(2 \pi)^{\frac{d}{2}}} \eta_{q}(y)\right)_{L^{2}\left(\mathbb{R}^{d+3}\right)}\right|
$$

from above by

$$
\frac{1}{(2 \pi)^{\frac{d+3}{2}}} \frac{1}{1-\|P\|_{\infty}}\left\|\phi-\phi_{n}\right\|_{L^{1}\left(\mathbb{R}^{d+3}\right)} \rightarrow 0, \quad n \rightarrow \infty
$$

by means of (3.1). Thus we arrive at the orthogonality relation

$$
\left(\phi(x, y), \frac{e^{i k x}}{(2 \pi)^{\frac{d}{2}}} \eta_{q}(y)\right)_{L^{2}\left(\mathbb{R}^{d+3}\right)}=0, \quad(k, q) \in S_{\sqrt{a}}^{d+3} \quad \text { a.e. },
$$

which by virtue of the part a) of Theorem 6 of [19] gives us that problem (1.6) possesses a unique solution $u(x, y) \in H^{2}\left(\mathbb{R}^{d+3}\right)$. By applying the generalized Fourier transform (1.17) to both sides of equations (1.6) and (1.7), we arrive at

$$
\tilde{\hat{u}}(k, q)=\frac{\tilde{\hat{\phi}}(k, q)}{k^{2}+q^{2}-a}, \quad \tilde{\hat{u}}_{n}(k, q)=\frac{\tilde{\hat{\phi}}_{n}(k, q)}{k^{2}+q^{2}-a}, \quad n \in \mathbb{N} .
$$


Let us introduce as a technical tool a spherical layer in the space of $d+3$ dimensions, namely

$$
B_{\sigma}:=\left\{(k, q) \in \mathbb{R}^{d+3} \mid \sqrt{a}-\sigma \leq \sqrt{k^{2}+q^{2}} \leq \sqrt{a}+\sigma\right\}, \quad 0<\sigma<\sqrt{a} .
$$

Then we can write the difference $\tilde{\hat{u}}_{n}(k, q)-\tilde{\hat{u}}(k, q)$ as

$$
\frac{\tilde{\hat{\phi}}_{n}(k, q)-\tilde{\hat{\phi}}(k, q)}{k^{2}+q^{2}-a} \chi_{B_{\sigma}}+\frac{\tilde{\hat{\phi}}_{n}(k, q)-\tilde{\hat{\phi}}(k, q)}{k^{2}+q^{2}-a} \chi_{B_{\sigma}^{c}} .
$$

The second term in (3.7) can be easily estimated from above in the absolute value by $\frac{\left|\tilde{\hat{\phi}}_{n}(k, q)-\tilde{\hat{\phi}}(k, q)\right|}{\sqrt{a} \sigma}$, which clearly gives us the upper bound for it in the $L^{2}\left(\mathbb{R}^{d+3}\right)$ norm as

$$
\frac{1}{\sqrt{a} \sigma}\left\|\phi_{n}-\phi\right\|_{L^{2}\left(\mathbb{R}^{d+3}\right)} \rightarrow 0, \quad n \rightarrow \infty
$$

as assumed in our theorem. For the purpose of investigating the first term in (3.7), we will rely on the identities

$$
\begin{gathered}
\tilde{\hat{\phi}}(k, q)=\tilde{\hat{\phi}}(\sqrt{a}, \omega)+\int_{\sqrt{a}}^{\sqrt{k^{2}+q^{2}}} \frac{\partial \tilde{\hat{\phi}}}{\partial s}(s, \omega) d s, \\
\tilde{\hat{\phi}}_{n}(k, q)=\tilde{\hat{\phi}}_{n}(\sqrt{a}, \omega)+\int_{\sqrt{a}}^{\sqrt{k^{2}+q^{2}}} \frac{\partial \tilde{\hat{\phi}}_{n}}{\partial s}(s, \omega) d s, \quad n \in \mathbb{N} .
\end{gathered}
$$

Orthogonality relations (1.19) and (3.6) imply that $\tilde{\hat{\phi}}_{n}(\sqrt{a}, \omega)$ and $\tilde{\hat{\phi}}(\sqrt{a}, \omega)$ vanish. Therefore, the first term in (3.7) can be estimated from above in the absolute value by

$$
\left\|\left(\nabla_{k}+\nabla_{q}\right)\left(\tilde{\hat{\phi}}_{n}(k, q)-\tilde{\hat{\phi}}(k, q)\right)\right\|_{L^{\infty}\left(\mathbb{R}^{d+3}\right)} \frac{\chi_{B_{\sigma}}}{\sqrt{a}},
$$

which gives us the upper bound for it in the $L^{2}\left(\mathbb{R}^{d+3}\right)$ norm as

$$
C\left\|\left(\nabla_{k}+\nabla_{q}\right)\left(\tilde{\hat{\phi}}_{n}(k, q)-\tilde{\hat{\phi}}(k, q)\right)\right\|_{L^{\infty}\left(\mathbb{R}^{d+3}\right)} \rightarrow 0, \quad n \rightarrow \infty
$$

by means of the part b) of Lemma 5 below. Thus, when $a>0$, we have

$$
\left\|u_{n}(x, y)-u(x, y)\right\|_{L^{2}\left(\mathbb{R}^{d+3}\right)}=\left\|\tilde{\hat{u}}_{n}(k, q)-\tilde{\hat{u}}(k, q)\right\|_{L^{2}\left(\mathbb{R}^{d+3}\right)} \rightarrow 0, \quad n \rightarrow \infty,
$$

such that via the part b) of Lemma 4 below $u_{n}(x, y) \rightarrow u(x, y)$ in $H^{2}\left(\mathbb{R}^{d+3}\right), n \rightarrow \infty$, which completes the proof of the part a) of the theorem.

\section{4.. Auxiliary results}

The trivial lemma below helps us to conclude the proofs of Theorems 2 and 3 by telling that it is sufficient to prove the convergence in $L^{2}$ of the solutions of the studied equations as $n \rightarrow \infty$.

Lemma 4. a) Let the conditions of Theorem 2 hold, such that $u(x, y), u_{n}(x, y) \in$ $H^{2}\left(\mathbb{R}^{6}\right)$ with $n \in \mathbb{N}$ are the unique solutions of equations (1.2) and (1.3) respectively and $u_{n}(x, y) \rightarrow u(x, y)$ in $L^{2}\left(\mathbb{R}^{6}\right)$ as $n \rightarrow \infty$. Then $u_{n}(x, y) \rightarrow u(x, y)$ in $H^{2}\left(\mathbb{R}^{6}\right)$ as $n \rightarrow \infty$.

b) Let the assumptions of Theorem 3 be valid, such that $u(x, y), u_{n}(x, y) \in H^{2}\left(\mathbb{R}^{d+3}\right)$ with $n \in \mathbb{N}$ and $d \in \mathbb{N}$ are the unique solutions of equations (1.6) and (1.7) respectively and $u_{n}(x, y) \rightarrow u(x, y)$ in $L^{2}\left(\mathbb{R}^{d+3}\right)$ as $n \rightarrow \infty$. Then $u_{n}(x, y) \rightarrow u(x, y)$ in $H^{2}\left(\mathbb{R}^{d+3}\right)$ as $n \rightarrow \infty$. 
Proof. a) Equations (1.2) and (1.3) with $a \geq 0$ along with the assumptions of Theorem 2 yield that the norm $\left\|\left(\Delta_{x}+\Delta_{y}\right)\left(u_{n}-u\right)\right\|_{L^{2}\left(\mathbb{R}^{6}\right)}$ is bounded above by

$$
\left\|f_{n}-f\right\|_{L^{2}\left(\mathbb{R}^{6}\right)}+\left(\|V\|_{L^{\infty}\left(\mathbb{R}^{3}\right)}+\|U\|_{L^{\infty}\left(\mathbb{R}^{3}\right)}+a\right)\left\|u_{n}-u\right\|_{L^{2}\left(\mathbb{R}^{6}\right)} \rightarrow 0, n \rightarrow \infty .
$$

By virtue of definition (1.4) we obtain $u_{n}(x, y) \rightarrow u(x, y)$ in $H^{2}\left(\mathbb{R}^{6}\right)$ as $n \rightarrow \infty$.

b) From equations (1.6) and (1.7) for $a \geq 0$ under the conditions of Theorem 3 we derive the estimate from above for the norm $\left\|\left(\Delta_{x}+\Delta_{y}\right)\left(u_{n}-u\right)\right\|_{L^{2}\left(\mathbb{R}^{d+3}\right)}$ given by

$$
\left\|\phi_{n}-\phi\right\|_{L^{2}\left(\mathbb{R}^{d+3}\right)}+\left(a+\|U\|_{L^{\infty}\left(\mathbb{R}^{3}\right)}\right)\left\|u_{n}-u\right\|_{L^{2}\left(\mathbb{R}^{d+3}\right)} \rightarrow 0, \quad n \rightarrow \infty .
$$

Thus, definition (1.4) implies that $u_{n}(x, y) \rightarrow u(x, y)$ in $H^{2}\left(\mathbb{R}^{d+3}\right)$ as $n \rightarrow \infty$.

The $L^{\infty}\left(\mathbb{R}^{6}\right)$ and $L^{\infty}\left(\mathbb{R}^{d+3}\right)$ norms studied in the following lemma are finite by virtue of Lemmas 11 and 12 of [19] respectively. We go further by showing that they converge to zero.

Lemma 5. a) Let the assumptions of Theorem 2 be valid. Then

$$
\left\|\left(\nabla_{k}+\nabla_{q}\right)\left[\tilde{\tilde{f}}_{n}(k, q)-\tilde{\tilde{f}}(k, q)\right]\right\|_{L^{\infty}\left(\mathbb{R}^{6}\right)} \rightarrow 0, n \rightarrow \infty .
$$

b) Let the conditions of Theorem 3 hold. Then we have

$$
\left\|\left(\nabla_{k}+\nabla_{q}\right)\left[\tilde{\hat{\phi}}_{n}(k, q)-\tilde{\hat{\phi}}(k, q)\right]\right\|_{L^{\infty}\left(\mathbb{R}^{d+3}\right)} \rightarrow 0, n \rightarrow \infty .
$$

Proof. To prove the part a) of the lemma, we need to estimate the quantity

$$
\left(\nabla_{k}+\nabla_{q}\right)\left[\tilde{\tilde{f}}_{n}(k, q)-\tilde{\tilde{f}}(k, q)\right]=\left(f_{n}(x, y)-f(x, y), \eta_{q}(y) \nabla_{k} \varphi_{k}(x)\right)_{L^{2}\left(\mathbb{R}^{6}\right)}+
$$

$$
+\left(f_{n}(x, y)-f(x, y), \varphi_{k}(x) \nabla_{q} \eta_{q}(y)\right)_{L^{2}\left(\mathbb{R}^{6}\right)} .
$$

By means of the Lippmann-Schwinger equations (1.12) and (1.13), we have

$$
\begin{aligned}
& \nabla_{k} \varphi_{k}(x)=\frac{e^{i k x}}{(2 \pi)^{\frac{3}{2}}} i x+(I-Q)^{-1} Q \frac{e^{i k x}}{(2 \pi)^{\frac{3}{2}}} i x+(I-Q)^{-1}\left(\nabla_{k} Q\right)(I-Q)^{-1} \frac{e^{i k x}}{(2 \pi)^{\frac{3}{2}}}, \\
& \nabla_{q} \eta_{q}(y)=\frac{e^{i q y}}{(2 \pi)^{\frac{3}{2}}} i y+(I-P)^{-1} P \frac{e^{i q y}}{(2 \pi)^{\frac{3}{2}}} i y+(I-P)^{-1}\left(\nabla_{q} P\right)(I-P)^{-1} \frac{e^{i q y}}{(2 \pi)^{\frac{3}{2}}} .
\end{aligned}
$$

Here the operators $\nabla_{k} Q, \nabla_{q} P: L^{\infty}\left(\mathbb{R}^{3}\right) \rightarrow L^{\infty}\left(\mathbb{R}^{3} ; \mathbb{C}^{3}\right)$ have the integral kernels

$$
\nabla_{k} Q(x, y, k):=-\frac{i}{4 \pi} e^{i|k||x-y|} \frac{k}{|k|} V(y), \quad \nabla_{q} P(y, z, q):=-\frac{i}{4 \pi} e^{i|q||y-z|} \frac{q}{|q|} U(z)
$$

respectively. Obviously, for the operator norms we have the following inequalities

$$
\left\|\nabla_{k} Q\right\|_{\infty} \leq \frac{1}{4 \pi}\|V\|_{L^{1}\left(\mathbb{R}^{3}\right)}<\infty, \quad\left\|\nabla_{q} P\right\|_{\infty} \leq \frac{1}{4 \pi}\|U\|_{L^{1}\left(\mathbb{R}^{3}\right)}<\infty
$$

by means of the rate of decay of the scalar potentials $V(x)$ and $U(y)$ given precisely in Assumption 1. Thus, in order to establish the convergence to zero as $n \rightarrow \infty$ of the $L^{\infty}\left(\mathbb{R}^{6}\right)$ norm of the expression (4.1), we will need to estimate the six terms given below. The first one is

$$
T_{1, n}(k, q):=\left(f_{n}(x, y)-f(x, y), \frac{e^{i k x}}{(2 \pi)^{\frac{3}{2}}} i x \eta_{q}(y)\right)_{L^{2}\left(\mathbb{R}^{6}\right)}, \quad k, q \in \mathbb{R}^{3} .
$$


We easily obtain via (2.4) that

$$
\left|T_{1, n}(k, q)\right| \leq \frac{1}{(2 \pi)^{3}} \frac{1}{1-\|P\|_{\infty}}\left\||x| f_{n}-|x| f\right\|_{L^{1}\left(\mathbb{R}^{6}\right)},
$$

such that $\left\|T_{1, n}(k, q)\right\|_{L^{\infty}\left(\mathbb{R}^{6}\right)} \rightarrow 0, n \rightarrow \infty$ by virtue of the assumptions of Theorem 2 . The second term which we need to estimate is given by

$$
T_{2, n}(k, q):=\left(f_{n}(x, y)-f(x, y),(I-Q)^{-1} Q \frac{e^{i k x}}{(2 \pi)^{\frac{3}{2}}} i x \eta_{q}(y)\right)_{L^{2}\left(\mathbb{R}^{6}\right)}, \quad k, q \in \mathbb{R}^{3} .
$$

Evidently, we can find the upper bound for it in the absolute value as

$$
\frac{1}{1-\|Q\|_{\infty}} \frac{1}{1-\|P\|_{\infty}}\left\{C\|V(x) x\|_{L^{\infty}\left(\mathbb{R}^{3}\right)}+C\|V(x) x\|_{L^{\frac{4}{3}\left(\mathbb{R}^{3}\right)}}\right\}\left\|f_{n}-f\right\|_{L^{1}\left(\mathbb{R}^{6}\right)},
$$

such that the norm $\left\|T_{2, n}(k, q)\right\|_{L^{\infty}\left(\mathbb{R}^{6}\right)} \rightarrow 0$ as $n \rightarrow \infty$ by means of the assumptions of Theorem 2 and (2.2). Note that in the argument above we used the upper bound for the norm $\left\|Q e^{i k x} x\right\|_{L^{\infty}\left(\mathbb{R}^{3}\right)}$ obtained in the proof of Lemma 2.4 of [18] along with (2.4). The third term we need to investigate is

$$
T_{3, n}(k, q):=\left(f_{n}(x, y)-f(x, y),(I-Q)^{-1}\left(\nabla_{k} Q\right)(I-Q)^{-1} \frac{e^{i k x}}{(2 \pi)^{\frac{3}{2}}} \eta_{q}(y)\right)_{L^{2}\left(\mathbb{R}^{6}\right)},
$$

with $k, q \in \mathbb{R}^{3}$. By virtue of (4.2) along with (2.4), we easily derive the inequality

$$
\left|T_{3, n}(k, q)\right| \leq \frac{1}{4 \pi(2 \pi)^{3}} \frac{\|V\|_{L^{1}\left(\mathbb{R}^{3}\right)}}{\left(1-\|P\|_{\infty}\right)\left(1-\|Q\|_{\infty}\right)^{2}}\left\|f_{n}-f\right\|_{L^{1}\left(\mathbb{R}^{6}\right)},
$$

such that the norm $\left\|T_{3, n}(k, q)\right\|_{L^{\infty}\left(\mathbb{R}^{6}\right)} \rightarrow 0$ as $n \rightarrow \infty$ by means of the conditions of Theorem 2 and (2.2). Let us take a look at the fourth term, which is

$$
R_{1, n}(k, q):=\left(f_{n}(x, y)-f(x, y), \varphi_{k}(x) \frac{e^{i q y}}{(2 \pi)^{\frac{3}{2}}} i y\right)_{L^{2}\left(\mathbb{R}^{6}\right)},
$$

where $k, q \in \mathbb{R}^{3}$. Using (2.4), we obtain the upper bound

$$
\left|R_{1, n}(k, q)\right| \leq \frac{1}{(2 \pi)^{3}} \frac{1}{1-\|Q\|_{\infty}}\left\||y| f_{n}-|y| f\right\|_{L^{1}\left(\mathbb{R}^{6}\right)} .
$$

Hence $\left\|R_{1, n}(k, q)\right\|_{L^{\infty}\left(\mathbb{R}^{6}\right)} \rightarrow 0, n \rightarrow \infty$ via the assumptions of Theorem 2 . The fifth term to be estimated is given by

$$
R_{2, n}(k, q):=\left(f_{n}(x, y)-f(x, y), \varphi_{k}(x)(I-P)^{-1} P \frac{e^{i q y}}{(2 \pi)^{\frac{3}{2}}} i y\right)_{L^{2}\left(\mathbb{R}^{6}\right)}, \quad k, q \in \mathbb{R}^{3} .
$$

Evidently, via (2.4) it can be bounded from above in the absolute value by

$$
\frac{1}{1-\|Q\|_{\infty}} \frac{1}{1-\|P\|_{\infty}}\left\{C\|U(y) y\|_{L^{\infty}\left(\mathbb{R}^{3}\right)}+C\|U(y) y\|_{L^{\frac{4}{3}\left(\mathbb{R}^{3}\right)}}\right\}\left\|f_{n}-f\right\|_{L^{1}\left(\mathbb{R}^{6}\right)},
$$

such that $\left\|R_{2, n}(k, q)\right\|_{L^{\infty}\left(\mathbb{R}^{6}\right)} \rightarrow 0$ as $n \rightarrow \infty$ by virtue of the conditions of Theorem 2 and (2.2). We used the estimate from above for the norm $\left\|P e^{i q y} y\right\|_{L^{\infty}\left(\mathbb{R}^{3}\right)}$ derived in the proof of Lemma 11 of [19]. Finally, it remains to investigate

$$
R_{3, n}(k, q):=\left(f_{n}(x, y)-f(x, y), \varphi_{k}(x)(I-P)^{-1}\left(\nabla_{q} P\right)(I-P)^{-1} \frac{e^{i q y}}{(2 \pi)^{\frac{3}{2}}}\right)_{L^{2}\left(\mathbb{R}^{6}\right)}
$$


with $k, q \in \mathbb{R}^{3}$. Bounds (2.4) and (4.2) imply

$$
\left|R_{3, n}(k, q)\right| \leq \frac{1}{4 \pi(2 \pi)^{3}} \frac{\|U\|_{L^{1}\left(\mathbb{R}^{3}\right)}}{\left(1-\|Q\|_{\infty}\right)\left(1-\|P\|_{\infty}\right)^{2}}\left\|f_{n}-f\right\|_{L^{1}\left(\mathbb{R}^{6}\right)} .
$$

Thus, the assumptions of Theorem 2 along with (2.2) yield $\left\|R_{3, n}(k, q)\right\|_{L^{\infty}\left(\mathbb{R}^{6}\right)} \rightarrow 0$ as $n \rightarrow \infty$, which completes the proof of the part a) of the lemma. Then we turn our attention to establishing the result of the part $b$ ).

Obviously, for $k \in \mathbb{R}^{d}$ and $q \in \mathbb{R}^{3}$, we write $\left(\nabla_{k}+\nabla_{q}\right) \tilde{\hat{\phi}}(k, q)$ as

$$
\left(\phi(x, y), \frac{e^{i k x}}{(2 \pi)^{\frac{d}{2}}} i x \eta_{q}(y)\right)_{L^{2}\left(\mathbb{R}^{d+3}\right)}+\left(\phi(x, y), \frac{e^{i k x}}{(2 \pi)^{\frac{d}{2}}} \nabla_{q} \eta_{q}(y)\right)_{L^{2}\left(\mathbb{R}^{d+3}\right)}
$$

and $\left(\nabla_{k}+\nabla_{q}\right) \tilde{\hat{\phi}}_{n}(k, q)$ as

$$
\left(\phi_{n}(x, y), \frac{e^{i k x}}{(2 \pi)^{\frac{d}{2}}} i x \eta_{q}(y)\right)_{L^{2}\left(\mathbb{R}^{d+3}\right)}+\left(\phi_{n}(x, y), \frac{e^{i k x}}{(2 \pi)^{\frac{d}{2}}} \nabla_{q} \eta_{q}(y)\right)_{L^{2}\left(\mathbb{R}^{d+3}\right)} .
$$

Therefore, to conclude the proof of the lemma it remains to investigate the four terms given below. The first one is

$$
M_{1, n}(k, q):=\left(\phi_{n}(x, y)-\phi(x, y), \frac{e^{i k x}}{(2 \pi)^{\frac{d}{2}}} i x \eta_{q}(y)\right)_{L^{2}\left(\mathbb{R}^{d+3}\right)}, \quad k \in \mathbb{R}^{d}, \quad q \in \mathbb{R}^{3},
$$

such that via (2.4)

$$
\left|M_{1, n}(k, q)\right| \leq \frac{1}{(2 \pi)^{\frac{d+3}{2}}} \frac{1}{1-\|P\|_{\infty}}\left\||x| \phi_{n}-|x| \phi\right\|_{L^{1}\left(\mathbb{R}^{d+3}\right)} .
$$

Hence $\left\|M_{1, n}(k, q)\right\|_{L^{\infty}\left(\mathbb{R}^{d+3}\right)} \rightarrow 0, n \rightarrow \infty$ due to the assumptions of Theorem 3 . The second expression is given by

$$
M_{2, n}(k, q):=\left(\phi_{n}(x, y)-\phi(x, y), \frac{e^{i k x}}{(2 \pi)^{\frac{d}{2}}} \frac{e^{i q y}}{(2 \pi)^{\frac{3}{2}}} i y\right)_{L^{2}\left(\mathbb{R}^{d+3}\right)}, \quad k \in \mathbb{R}^{d}, \quad q \in \mathbb{R}^{3}
$$

Hence

$$
\left|M_{2, n}(k, q)\right| \leq \frac{1}{(2 \pi)^{\frac{d+3}{2}}}\left\||y| \phi_{n}-|y| \phi\right\|_{L^{1}\left(\mathbb{R}^{d+3}\right)}
$$

and therefore, $\left\|M_{2, n}(k, q)\right\|_{L^{\infty}\left(\mathbb{R}^{d+3}\right)} \rightarrow 0, n \rightarrow \infty$ by virtue of one of the conditions of Theorem 3. The third term to be estimated for $k \in \mathbb{R}^{d}, q \in \mathbb{R}^{3}$ will be

$$
M_{3, n}(k, q):=\left(\phi_{n}(x, y)-\phi(x, y), \frac{e^{i k x}}{(2 \pi)^{\frac{d}{2}}}(I-P)^{-1} P \frac{e^{i q y}}{(2 \pi)^{\frac{3}{2}}} i y\right)_{L^{2}\left(\mathbb{R}^{d+3}\right)} .
$$

It can be bounded above in the absolute value by by

$$
\frac{1}{1-\|P\|_{\infty}}\left\{C\|U(y) y\|_{L^{\infty}\left(\mathbb{R}^{3}\right)}+C\|U(y) y\|_{L^{\frac{4}{3}\left(\mathbb{R}^{3}\right)}}\right\}\left\|\phi_{n}-\phi\right\|_{L^{1}\left(\mathbb{R}^{d+3}\right)},
$$

such that the norm $\left\|M_{3, n}(k, q)\right\|_{L^{\infty}\left(\mathbb{R}^{d+3}\right)} \rightarrow 0$ as $n \rightarrow \infty$ by means of the assumptions of Theorem 3 and (3.1). When obtaining the estimate above, we relied on the upper bound for 
the norm $\left\|P e^{i q y} y\right\|_{L^{\infty}\left(\mathbb{R}^{3}\right)}$ derived in the proof of Lemma 11 of [19]. Finally, we consider $M_{4, n}(k, q)$, defined for $k \in \mathbb{R}^{d}, q \in \mathbb{R}^{3}$ as

$$
\left(\phi_{n}(x, y)-\phi(x, y), \frac{e^{i k x}}{(2 \pi)^{\frac{d}{2}}}(I-P)^{-1}\left(\nabla_{q} P\right)(I-P)^{-1} \frac{e^{i q y}}{(2 \pi)^{\frac{3}{2}}}\right)_{L^{2}\left(\mathbb{R}^{d+3}\right)},
$$

such that via (4.2)

$$
\left|M_{4, n}(k, q)\right| \leq \frac{C}{\left(1-\|P\|_{\infty}\right)^{2}}\|U\|_{L^{1}\left(\mathbb{R}^{3}\right)}\left\|\phi_{n}-\phi\right\|_{L^{1}\left(\mathbb{R}^{d+3}\right)}
$$

and $\left\|M_{4, n}(k, q)\right\|_{L^{\infty}\left(\mathbb{R}^{d+3}\right)} \rightarrow 0$ as $n \rightarrow \infty$ due to the conditions of Theorem 3 and (3.1), which completes the proof of the part b) of the lemma.

\section{References}

[1] C. Amrouche, V. Girault, J. Giroire, Dirichlet and Neumann exterior problems for the n-dimensional Laplace operator: an approach in weighted Sobolev spaces, J. Math. Pures Appl. (9), 76 (1997), No.1, 55-81.

[2] C. Amrouche, F. Bonzom, Mixed exterior Laplace's problem, J. Math. Anal. Appl., 338 (2008), No.1, 124140.

[3] P. Bolley, T.L. Pham, Propriétés d'indice en théorie Holderienne pour des opérateurs différentiels elliptiques dans $R^{n}$, J. Math. Pures Appl. (9), 72 (1993), No.1, 105-119.

[4] P. Bolley, T.L. Pham, Propriété d'indice en théorie Hölderienne pour le problème extérieur de Dirichlet, Comm. Partial Differential Equations, 26 (2001), No. 1-2, 315-334.

[5] N. Benkirane, Propriété d'indice en théorie holderienne pour des opérateurs elliptiques dans $R^{n}$, CRAS, 307, Série I (1988), No.11, 577-580.

[6] H.L. Cycon, R.G. Froese, W. Kirsch, B. Simon, Schrödinger Operators with Application to Quantum Mechanics and Global Geometry, Springer-Verlag, Berlin (1987).

[7] A. Ducrot, M. Marion, V. Volpert, Systemes de réaction-diffusion sans propriété de Fredholm, CRAS, 340 (2005), No.9, 659-664.

[8] A. Ducrot, M. Marion, V. Volpert, Reaction-diffusion problems with non Fredholm operators, Advances Diff. Equations, 13 (2008), No. 11-12, 1151-1192.

[9] M. Goldberg, W. Schlag, A limiting absorption principle for the three-dimensional Schrödinger equation with $L^{p}$ potentials, Int. Math. Res. Not., (2004), No. 75, 4049-4071.

[10] B.L.G. Jonsson, M. Merkli, I.M. Sigal, F. Ting, Applied Analysis, In preparation.

[11] T. Kato, Wave operators and similarity for some non-selfadjoint operators, Math. Ann., 162 (1965/1966), 258-279.

[12] E. Lieb, M. Loss, Analysis. Graduate Studies in Mathematics, 14, American Mathematical Society, Providence (1997).

[13] D.E. Pelinovsky, J. Yang, A normal form for nonlinear resonance of embedded solitons, R. Soc. Lond. Proc. Ser. A Math. Phys. Eng. Sci., 458 (2002), No. 2022, 1469-1497.

[14] M. Reed, B. Simon, Methods of Modern Mathematical Physics, III: Scattering Theory, Academic Press (1979).

[15] I. Rodnianski, W. Schlag, Time decay for solutions of Schrödinger equations with rough and time-dependent potentials, Invent. Math., 155 (2004), No. 3, 451-513.

[16] V. Volpert. Elliptic partial differential equations. Volume 1. Fredholm theory of elliptic problems in unbounded domains. Birkhauser, 2011.

[17] V. Volpert, B. Kazmierczak, M. Massot, Z.Peradzynski, Solvability conditions for elliptic problems with non-Fredholm operators, Appl.Math., 29 (2002), No. 2, 219-238.

[18] V. Vougalter, V. Volpert, Solvability conditions for some non Fredholm operators, Proc. Edinb. Math. Soc. (2), 54 (2011), No.1, 249-271

[19] V. Vougalter, V. Volpert. On the solvability conditions for some non Fredholm operators, Int. J. Pure Appl. Math., 60 (2010), No. 2, 169-191.

[20] V. Vougalter, V. Volpert. On the solvability conditions for the diffusion equation with convection terms, Commun. Pure Appl. Anal., 11 (2012), No. 1, 365-373. 
[21] V. Vougalter, V. Volpert. Solvability relations for some non Fredholm operators, Int. Electron. J. Pure Appl. Math., 2 (2010), No. 1, 75-83.

[22] V. Volpert, V. Vougalter. On the solvability conditions for a linearized Cahn-Hilliard equation, Rend. Istit. Mat. Univ. Trieste, 43 (2011), 1-9.

[23] V. Vougalter, V. Volpert. Solvability conditions for a linearized Cahn-Hilliard equation of sixth order, Math. Model. Nat. Phenom., 7 (2012), No. 2, 146-154.

[24] V. Vougalter, V. Volpert. Solvability conditions for some linear and nonlinear non-Fredholm elliptic problems, Anal. Math. Phys., 2 (2012), No.4, 473-496.

[25] V. Volpert, V. Vougalter. Solvability in the sense of sequences to some non-Fredholm operators, Electron. J. Differential Equations 2013, No.160, 16pp.

Department of Mathematics and Applied Mathematics, University of Cape Town, PriVATE BAG X1, Rondebosch 7701, South AFricA

E-mail address: vitali@math.toronto.edu

Institute CAmille JoRdan, UMR 5208 CNRS, University LyON 1, VilleurbanNe, 69622, FRANCE

E-mail address: volpert@math. univ-lyonl.fr 